\title{
Concentration and Persistence of Bacilli in the Fingers and Toes of Patients with Lepromatous Leprosy
}

\author{
S. HIRAMALINI*, N. A. JOSEPH† AND C. J. G. CHACKO \\ Schieffelin Leprosy Research \& Training Centre, Karigiri, North Arcot Dist., Tamil Nadu, \\ India
}

\begin{abstract}
In 41 patients with lepromatous leprosy, the fingers and toes were found to be the site with the highest bacillary load, the fingers being more productive than the earlobe or buttock and the toes being more productive than the buttock. Neither was the bacillary index at the finger significantly different from that at the toe, nor was the bacillary index at the terminal phalanx significantly different from that at the middle phalanx in either the finger or the toe. However, the terminal phalanx of the finger harboured more solid bacilli than the middle phalanx. In 14 long-treated low index cases where BI had registered a fall, and was not more than $2+$ at any of the routine smear sites, the fingers and toes harboured more bacilli than the earlobe. In one long treated smear negative case, the terminal phalanges of the fingers and toes proved to be the only skin sites positive for bacilli, all other routine sites, being acid-fast bacilli negative.
\end{abstract}

\section{Introduction}

Ridley and Jopling have reported that the fingers were the skin sites with the greatest bacterial load and the greatest number of solid staining bacilli following a study on 30 patients with lepromatous leprosy (Ridley et al., 1976). It was decided to see whether this concentration of bacilli held good in tropical India as well. They had studied toes only in 8 cases. Since closed footwear is hardly used in India, it was decided to study toes as well, hoping to compare the bacillary load at fingers to that at toes in barefoot patients.

\section{Material and Methods}

Forty-one patients with lepromatous leprosy from the Schieffelin Leprosy Research \& Training Centre, Karigiri, were studied at random. All of them had treatment for periods ranging from 6 months to 20 years. Surprisingly we India.

* Final year medical student, Christian Medical College, Vellore, Tamil Nadu,

† Technician, Smear Laboratory, Schieffelin Leprosy Research \& Training Centre, Karigiri, North Arcot Dist., Tamil Nadu, India.

$\ddagger$ Head, Radda Barnen Research Laboratories, Schieffelin Leprosy Research \& Training Centre, Karigiri, and Professor of Pathology, Christian Medical College and Hospital, Vellore, North Arcot Dist., Tamil Nadu, India.

Received for publication 11 May, 1978.

0305-7518/78/0901-0223\$01.00/0

๑ 1978 British Leprosy Relief Association 
found no barefoot. patients. All patients studied wore the open type of footwear, i.e. sandals or slippers that are closed at the sides but leave the toetips exposed. Smears were taken from routine sites such as right ear-lobe, left forehead, right chin, and left buttock. Nasal mucosa was also studied, but as part of another project. It has not been included as a routine site in statistical calculations in this study. The special sites studied were:

(a) dorsum of the middle and terminal phalanges of the middle finger of both hands;

(b) dorsum of the middle and terminal phalanges of the second toe of both feet.

In either case (1) if there was a clinical lesion on the digit or (2) if the digit was missing, the adjacent digit was studied (3rd toe in the case of feet). The modified Ziehl-Neelsen method was used for staining; BI was estimated according to Ridley's logarithmic scale (Ridley, 1964) and MI according to the criteria of Waters and Rees (Waters and Rees, 1962).

On finding that more bacilli were found at the fingers and toes as compared to accepted sites such as earlobe and buttock, it was decided to see whether the fingers and toes harboured bacilli in greater numbers in long-treated cases where the BI at routine sites had registered a fall. We arbitrarily classified those with a maximal bacillary load not exceeding $2+$ at any of the routine sites as low index cases, and compared the bacterial load at fingers and toes to that at routine sites in those cases. Fourteen such cases were studied, all having had treatment for 3 years or more. We also studied 8 long-treated smear negative cases at routine and special sites to check for persistence of bacilli, if any, at the fingers or toes.

\section{Results}

The results of the initial random study are summarized in Table 1. BACTERIAL INDEX RESULTS

(a) The maximal $\mathrm{BI}$ at the fingers was significantly greater than the maximal $\mathrm{BI}$ at the earlobes, the $\mathrm{BI}$ at the middle phalanx being greater than that at the earlobe $(P<0.02)$, and the $\mathrm{BI}$ at the terminal phalanx also being greater than that at the earlobe $(P<0.05)$.

(b) The maximal $\mathrm{BI}$ at the fingers was significantly greater than the maximal $\mathrm{BI}$ at the buttock; the $\mathrm{BI}$ at middle phalanx being greater than that at the buttock $(P<0.001)$, and the $\mathrm{BI}$ at the terminal phalanx also being greater than that at the buttock $(P<0.01)$.

(c) In 35 out of 39 cases, the finger was more productive than the nasal mucosa (in 2 cases nasal mucosa could not be studied).

(d) In 26 out of 41 cases the finger was more productive than the forehead and in 29 out of 41 cases, more productive than the chin.

(e) The maximal BI at toe was significantly greater than the maximal BI at buttock, the $\mathrm{BI}$ at middle phalanx being greater than that at the buttock $(P<0.01)$, and the $\mathrm{BI}$ at terminal phalanx being greater than that at the buttock $(P<0.02)$. 
TABLE 1

Bacterial and morphological indices of patients by routine sites, finger and toe

\begin{tabular}{|c|c|c|c|c|c|c|c|c|c|c|c|c|c|c|c|}
\hline \multirow[t]{2}{*}{ Patient } & \multicolumn{2}{|c|}{ Earlobe } & \multicolumn{2}{|c|}{ Forehead } & \multicolumn{2}{|c|}{ Chin } & \multicolumn{2}{|c|}{ Buttock } & \multicolumn{2}{|c|}{ Finger } & \multicolumn{2}{|c|}{ Toe } & \multicolumn{2}{|c|}{$\begin{array}{c}\text { Nasal } \\
\text { mucosa }\end{array}$} & \multirow{2}{*}{$\begin{array}{l}\text { Duration } \\
\text { of } \\
\text { treatment } \\
\text { (years) }\end{array}$} \\
\hline & BI & MI & $\mathrm{BI}$ & MI & BI & MI & BI & MI & BI & MI & BI & MI & BI & MI & \\
\hline 1 & 6 & 0.5 & 5 & 0.5 & 4 & 0 & 4 & 0 & 5 & 1 & 5 & 0 & 2 & 0 & 2.5 \\
\hline 2 & 4 & 0 & 4 & 0 & 3 & 0 & 3 & 0.5 & 4 & 0.5 & 4 & 0.5 & 1 & 0 & 10 \\
\hline 3 & 3 & 0 & 3 & 0 & 5 & 0 & 3 & 0 & 4 & 0 & 4 & 0 & 1 & 0 & 10 \\
\hline 4 & 3 & 0 & 3 & 0 & 2 & 0 & 2 & 0 & 4 & 0 & 3 & 0 & 4 & 0 & 1 \\
\hline 5 & 4 & 1 & 4 & 0 & 4 & 0 & 4 & 0 & 6 & 1 & 4 & 0.5 & 5 & 1.5 & 8 \\
\hline 6 & 3 & 0.5 & 3 & 0.5 & 3 & 0.5 & 3 & 0.5 & 4 & 1.5 & 5 & 2 & 2 & 0 & 13 \\
\hline 7 & 6 & 0 & 5 & 1 & 4 & 1 & 4 & 1 & 6 & 1.5 & 5 & 1 & 1 & 1 & 7 \\
\hline 8 & 5 & 0 & 4 & 0 & 3 & 0.5 & 4 & 0 & 6 & 0 & 5 & 0.5 & 0 & 0 & 2.5 \\
\hline 9 & 3 & 0 & 2 & 0 & 1 & 0 & 3 & 0 & 5 & 0 & 3 & 0 & 0 & 0 & 7 \\
\hline 10 & 0 & 0 & 0 & 0 & 0 & 0 & 0 & 0 & 0 & 0 & 0 & 0 & 0 & 0 & 15 \\
\hline 11 & 0 & 0 & 1 & 0 & 0 & 0 & 0 & 0 & 0 & 0 & 0 & 0 & 0 & 0 & 20 \\
\hline 12 & 2 & 0 & 3 & 0 & 5 & 0 & 4 & 0 & 4 & 0 & 4 & 0.5 & 0 & 0 & 9 \\
\hline 13 & 4 & 0 & 4 & 0 & 4 & 0 & 4 & 0 & 4 & 0 & 4 & 0 & 2 & 0 & 1 \\
\hline 14 & 0 & 0 & 0 & 0 & 0 & 0 & 0 & 0 & 2 & 0 & 2 & 0 & 0 & 0 & 20 \\
\hline 15 & 1 & 0 & 0 & 0 & 0 & 0 & 1 & 0 & 2 & 0 & 2 & 0 & 2 & 0 & 3 \\
\hline 16 & 2 & 0 & 1 & 0 & 0 & 0 & 0 & 0 & 3 & 0 & 3 & 0 & 0 & 0 & 4 \\
\hline 17 & 0 & 0 & 0 & 0 & 0 & 0 & 2 & 0 & 1 & 0 & 1 & 0 & 0 & 0 & 12 \\
\hline 18 & 1 & 0 & 0 & 0 & 1 & 0 & 1 & 0 & 1 & 0 & 0 & 0 & 0 & 0 & 3 \\
\hline 19 & 1 & 0 & 1 & 0 & 2 & 0 & 1 & 0 & 2 & 0 & 3 & 0 & 1 & 0 & 20 \\
\hline 20 & 3 & 0 & 3 & 0 & 2 & 0 & 3 & 0 & 3 & 0 & 4 & 0 & 0 & 0 & 1.5 \\
\hline 21 & 4 & 0 & 4 & 0 & 3 & 1 & 3 & 1 & 4 & 0.5 & 5 & 3 & 3 & 0 & 4 \\
\hline 22 & 1 & 0 & 0 & 0 & 0 & 0 & 1 & 0 & 2 & 0 & 1 & 0 & 0 & 0 & 5 \\
\hline 23 & 6 & 0 & 6 & 1.5 & 3 & 0 & 4 & 0 & 4 & 0 & 3 & 1 & 3 & 0 & 12 \\
\hline 24 & 4 & 0 & 4 & 1.5 & 4 & 0 & 3 & 0 & 3 & 0 & 3 & 1 & 0 & 0 & 0.5 \\
\hline 25 & 3 & 0 & 2 & 0 & 2 & 0 & 2 & 0 & 5 & 0.5 & 3 & 0 & 1 & 0 & 4 \\
\hline 26 & 4 & 0 & 4 & 0 & 5 & 0 & 3 & 0 & 5 & 0 & 4 & 0 & 2 & 0 & 4 \\
\hline 27 & 5 & 0 & 5 & 0 & 5 & 0 & 4 & 0 & 4 & 0 & 5 & 0.5 & 2 & 0 & 5 \\
\hline 28 & 4 & 0 & 3 & 0 & 3 & 0 & 1 & 0 & 4 & 0 & 2 & 0 & 2 & 0 & 2 \\
\hline 29 & 2 & 0 & 3 & 0 & 2 & 0 & 0 & 0 & 3 & 0 & 2 & 0 & 0 & 0 & 3 \\
\hline 30 & 0 & 0 & 1 & 0 & 1 & 0 & 1 & 0 & 2 & 0 & 1 & 0 & 0 & 0 & 15 \\
\hline 31 & 6 & 1 & 4 & 0 & 4 & 0 & 3 & 0 & 4 & 0 & 6 & 1 & 3 & 1.5 & 2 \\
\hline 32 & 2 & 0 & 2 & 0 & 2 & 0 & 1 & 0 & 4 & 0 & 5 & 0 & 0 & 0 & 16 \\
\hline 33 & 2 & 0 & 3 & 0 & 2 & 0 & 4 & 0 & 3 & 0 & 3 & 0 & 0 & 0 & 5 \\
\hline 34 & 1 & 0 & 1 & 0 & 1 & 0 & 2 & 0 & 2 & 0 & 2 & 0 & 0 & 0 & 6 \\
\hline 35 & 2 & 0 & 2 & 0 & 1 & 0 & 2 & 0 & 0 & 0 & 1 & 0 & - & - & 4 \\
\hline 36 & 2 & 0 & 2 & 0 & 1 & 0 & 2 & 0 & 3 & 0 & 3 & 0 & - & - & 5 \\
\hline 37 & 3 & 0 & 3 & 0 & 2 & 0 & 4 & 0 & 4 & 0 & 2 & 0 & 0 & 0 & 1 \\
\hline 38 & 5 & 0 & 4 & 0 & 4 & 0 & 4 & 0 & 5 & 1 & 3 & 0 & 0 & 0 & 3 \\
\hline 39 & 3 & 1 & 4 & 0 & 4 & 0 & 4 & 0 & 5 & 0.5 & 4 & 0.5 & 2 & 0 & 12 \\
\hline 40 & 3 & 0 & 3 & 0 & 2 & 0 & 3 & 0 & 3 & 0 & 3 & 0 & 1 & 0 & 5 \\
\hline 41 & 4 & 0 & 3 & 0 & 2 & 0 & 3 & 0 & 4 & 0 & 3 & 0 & 0 & 0 & 4 \\
\hline
\end{tabular}

(f) The toes were more productive than the forehead in 18 out of 41 cases, and as highly productive as the forehead in 15 out of 41 cases.

(g) The toes were more productive than the chin in 23 out of 41 cases, and more productive than the nasal mucosa in 31 out of 39 cases.

(h) Five per cent of the patients who were acid-fast bacilli negative at the earlobe and buttock showed bacilli at the fingers and toes. 
When BI at earlobe and buttock were low, the fingers and toes showed a greater bacillary load.

No statistically significant difference was observed between the average or maximal BI at fingers or toes as compared to the same at routine sites. However, while the routine site with maximal BI varied from patient to patient the fingers and toes proved to be sites with bacillary loads as high as that at the best routine site in 32 out of 41 cases. The BI at the fingers was not significantly different from that at the toes. The BI at the terminal phalanx was also not significantly greater than that at the middle phalanx in either the finger or toe.

\section{MORPHOLOGICAL INDEX RESULTS}

(a) The maximal $\mathrm{MI}$ at fingers was significantly greater than that at the buttock $(P<0.05)$. However, it was not significantly greater than that at the earlobe.

(b) The maximal $\mathrm{MI}$ at the terminal phalanx of the finger was significantly greater than the maximal MI at the middle phalanx, of the finger $(P<0.05)$. However such a difference did not exist in the case of toes.

(c) The maximal MI at the toes was significantly greater than the maximal MI at the earlobe $(P<0.05)$.

(d) The maximal MI at the toes was also significantly greater than the maximal MI at the buttock $(P<0.01)$.

(e) However, no significant difference was seen between the average or maximal MI at finger or toe when compared to the same at routine sites. The MI at finger was not significantly different from that at the toe.

\section{RESULTS FOR THE 14 LOW INDEX CASES}

(a) In 10 out of 14 cases the finger was more productive than the earlobe.The maximal BI obtained at the finger was significantly greater than that at the earlobe $(P<0.05)$.

TABLE 2

\begin{tabular}{lcc}
\hline Special site & BI & MI \\
\hline Right middle finger, middle phalanx & 0 & 0 \\
Left middle finger, middle phalanx & 0 & 0 \\
Right second toe, middle phalanx & 0 & 0 \\
Left second toe, middle phalanx & 0 & 0 \\
Right middle finger, terminal phalanx & $2+$ & 0 \\
Left middle finger, terminal phalanx & $1+$ & 0 \\
Right second toe, terminal phalanx & $2+$ & 0 \\
Left second toe, terminal phalanx & 0 & 0 \\
Nasal mucosa & 0 & 0 \\
\hline
\end{tabular}

Routine sites were all acid-fast bacilli negative.

The 8 smear negative cases studied for persistence of bacilli at routine sites, fingers and toes were AFB-negative at all sites. 
(b) In 11 out of 14 cases, the finger was more productive than the forehead.

(c) In 9 out of 14 cases the finger was more productive than the chin.

(d) In 9 out of 12 cases the finger was more productive than the nasal mucosa.

(e) The toe was more productive than the earlobe in 9 out of 14 cases. The maximal BI obtained at the toe was significantly greater than the maximal $\mathrm{BI}$ at the earlobe $(P<0.05)$.

(f) The toe was more productive than the forehead in 9 out of 14 cases and more productive than the chin in 9 out of 14 cases.

(g) The toe was more productive than the nasal mucosa in 8 out of 12 cases.

(h) However no statistically significant difference was got between the BI's at toe and buttock. Also the average or maximal BI at finger or toe was not significantly different from that at the routine sites. But while the routine site with maximal BI varied from person to person, the finger and toe were sites consistently as highly productive for bacilli as the best routine site in 11 out of 14 cases.

\section{CASE NO. 14}

This case merits special mention because here the fingers and toes were the only skin sites positive for acid-fast bacilli, all other routine skin sites being acid-fast bacilli negative. The patient is a 27-year-old male with a 15-year history of disease on regular treatment for 10 years. His BI had registered a progressive fall with treatment. In 1967 his average BI had been 3.62 and it fell to 0.75 in 1974 . No smears were done after that until 1977 when on routine smear examination he was found to be AFB-negative. However, on routine and special site smear studies, we got the results given in Table 2.

\section{Discussion}

Our findings are similar to those of Ridley and Jopling. Comparing the bacillary load at each routine skin-smear site including nasal mucosa to that at the finger, it was found that the finger harboured significantly greater numbers of bacilli than all these sites. Similarly, the toes proved more productive than the chin, buttock and nasal mucosa. Besides this, in long-treated cases, bacilli were found to persist in significantly greater numbers at the fingers and toes as compared to the earlobe, forehead, chin and nasal mucosa. These findings argue for the inclusion of finger skin smears on a routine basis for the bacteriological diagnosis of leprosy, assessment of progress following treatment, and finally before declaring a patient AFB-negative. The middle phalanx of the finger gave a BI as good as the terminal phalanx, but the MI at the terminal phalanx was significantly greater than that at the middle phalanx. This further suggests that the dorsum of the terminal phalanx of the finger be the site for skin smears.

The epidemiological significance of these findings is not known. Pedley, following his "contact smear" study (Pedley, 1970) has shown that bacilli probably do not emerge through intact skin. Even so, the high incidence of ulceration due to the vulnerability of the constantly used anaesthetic finger, 
promises a fair amount of infective potential to the lepromatous finger, so rich in solid-staining bacilli.

Just why the bacilli should concentrate in the fingers is not known. Thermographic studies by Hastings (1968), and Anish (1971), have shown that bacilli are present in greater numbers in the cooler areas of the body. Anish reports a relatively higher bacillary load in the cool forearm as compared to the warm axilla and scalp in patients with lepromatous leprosy. That the MI at the terminal phalanx was greater than that at the middle phalanx in the finger suggests that there may be a temperature gradient across the phalanges. A lower drug concentration at the fingertips consequent to a lesser perfusion as a result of disuse atrophy, and probably ulceration and fibrosis could be another reason for a high and persisting bacillary load in these areas. Such a decreased perfusion would make the finger a cooler site also. However, only thermographic studies can give a final answer. The rich nerve supply of the finger may be another reason why the neurotropic bacilli are present there in such large numbers.

But why the toes, which have an equally rich nerve supply and suffer similar trophic changes should register a relatively low BI is another poser. But then, none of the patients studied were barefoot. Even with the open type of footwear, air circulation is restricted and constant contact with the same surface is bound to generate a certain amount of frictional heat, all of which would go to make the toes not-so-cool a site. Still it is noteworthy that there were more solid bacilli in the toes than at the earlobe or buttock.

An important finding was that in at least one patient, who was AFBnegative at routine sites, we found bacilli persisting in the fingers and toes. It is striking that all the bacilli detected were at the terminal phalanges, the middle phalanges being AFB-negative. Perhaps like the nerve and Dartos muscle, which are proven sites of bacillary persistence, the terminal phalanx harbours persisters as well. Such persisters might play an important role in relapse in lepromatous patients.

Our not finding AFB in the fingers or toes of the 8 smear-negative cases does not necessarily go against this hypothesis since this study has all the disadvantages of a cross-sectional study. Perhaps the persisting bacilli if any had also been eradicated. The ideal way of studying persistence would be to do skin smears from routine sites and fingers and toes at intervals following initiation of therapy in patients with lepromatous leprosy and to check for persistence of bacilli in large numbers at the fingers and toes while the routine sites registered a fall in BI. We intend following up this patient to see whether he is heading for a relapse. If so, at least in his case, the terminal phalanges of the fingers and toes were the skin sites at which the bacilli persisted.

\section{Acknowledgements}

We are indebted to Mr Muthuratnam of the Department of Biostatistics, Christian Medical College and Hospital, Vellore, for analysis of the data and to $\mathrm{Mr}$ J. Ramamurthy for his secretarial help. We are also grateful to $\mathrm{Mr} \mathrm{K}$. Vilwanathan for his assistance in carrying out the study. 


\section{References}

Anish, S. A. (1971). The relationship between surface temperature and dermal invasion in lepromatous leprosy. Int. J. Lepr. 39, 848.

Hastings, R. C. et al. (1968). Bacterial density in skin in lepromatous leprosy as related to temperature. Lepr. Rev. 39, 71.

Pedley, J. C. (1970). Composite skin contact smears; a method demonstrating the non emergence of M. leprae from intact lepromatous skin. Lepr. Rev. 41, 31.

Ridley, D. S. (1964). Bacterial Indices in Leprosy in Theory and Practice, 2nd edit., pp. 620-622. R. G. Cochrane and T. F. Davey, Eds. John Wright and Sons, Ltd., Bristol.

Ridley, M., Jopling, W. H. and Ridley, D. S. (1976). Acid fast bacilli in the fingers of long treated lepromatous patients. Lepr. Rev. 47, 93.

Waters, M. F. R. and Rees, R. J. W. (1962). Changes in the morphology of M. leprae in patients under treatment. Int. J. Lepr. 30, 266. 